\title{
Early surgical therapy of infective endocarditis in children: A 15-year experience
}

\author{
Pirouz Shamszad, MD, ${ }^{a}$ Muhammad S. Khan, MD, ${ }^{b}$ Joseph W. Rossano, MD, ${ }^{c}$ and \\ Charles D. Fraser, Jr, MD ${ }^{\mathrm{b}}$
}

\begin{abstract}
Objectives: Infective endocarditis is rare in children but potentially carries high mortality and morbidity. Few data exist regarding surgical therapy and the associated outcomes in children with infective endocarditis. The aim of the present study was to describe the characteristics and outcomes of children undergoing surgery for infective endocarditis.
\end{abstract}

\begin{abstract}
Methods: A retrospective review of all patients aged 21 years or younger diagnosed with definitive infective endocarditis at a single center from 1996 to 2010 was performed.

Results: Of 76 identified patients with infective endocarditis (median age, 8.3 years; $73.9 \%$ boys), 46 patients (61\%) required surgical intervention. Staphylococcus aureus was most commonly isolated (18 patients, 24\%) followed by Streptococcus (17 patients, $22 \%$ ). Common surgical indications included severe valvular insufficiency in 13 patients, septic embolization in 12, concomitant severe valvular insufficiency and ventricular dysfunction in 9, persistent vegetations in 9 , and persistent bacteremia in 3 . Although early surgery was performed within 7 days of diagnosis in 35 patients (76\%), 25 (54\%) underwent surgery within 3 days or less. The factors associated with surgery included the presence of ventricular dysfunction, left-sided vegetation, severe valvular insufficiency, septic embolization, and $S$ aureus. Surgery within 3 days or less was associated with the presence of ventricular dysfunction and $S$ aureus. Native valve repair was performed in $50 \%$ of patients with native-valve disease. Postoperatively, no septic embolization events occurred and recurrence was low (2\%). The 1-, 5-, and 10 -year survival was $98 \% \pm 2 \%, 90 \% \pm 8 \%$, and $81 \% \pm 11 \%$, respectively.
\end{abstract}

Conclusions: Children with infective endocarditis can undergo successful early surgical therapy with a low risk of septic embolization, recurrence, and operative mortality. (J Thorac Cardiovasc Surg 2013;146:506-11)

Infective endocarditis (IE), although relatively uncommon in children, ${ }^{1,2}$ is a serious infection associated with significant morbidity and mortality. ${ }^{1-3}$ Effective treatment of IE typically involves prolonged medical therapy with intravenous antibiotics, which can be combined with surgical therapy in some patients. ${ }^{4-8}$ Often in complicated IE, the decision between surgical versus medical therapy can be complex.

The presence of congenital heart disease represents a significant difference between adult and pediatric populations and affects the epidemiology, treatment, and outcomes of IE. 1,3,9-11 Although surgical indications for IE have been

From the Lillie Frank Abercrombie Section of Cardiology, ${ }^{\mathrm{a}}$ Department of Pediatrics, Texas Children's Hospital, Baylor College of Medicine, Houston, Tex; Division of Congenital Heart Surgery, ${ }^{\mathrm{b}}$ Michael E. DeBakey Department of Surgery, Texas Children's Hospital Baylor College of Medicine, Houston, Tex; and Cardiac Center, ${ }^{\mathrm{c}}$ Department of Pediatrics, Children's Hospital of Philadelphia, University of Pennsylvania, Philadelphia, $\mathrm{Pa}$.

Disclosures: Authors have nothing to disclose with regard to commercial support.

Received for publication May 10, 2012; revisions received Sept 14, 2012; accepted for publication Dec 4, 2012; available ahead of print Jan 10, 2013

Address for reprints: Pirouz Shamszad, MD, Lillie Frank Abercrombie Section of Cardiology, Department of Pediatrics, Texas Children's Hospital, Baylor College of Medicine, 6621 Fannin St, MC-19345-C, Houston, TX 77030 (E-mail: pirouzshamszad@gmail.com).

$0022-5223 / \$ 36.00$

Copyright (c) 2013 by The American Association for Thoracic Surgery

http://dx.doi.org/10.1016/j.jtcvs.2012.12.001 outlined by the American Heart Association ${ }^{12}$ and endorsed by the American Academy of Pediatrics, the recommendations were determined from adult data, and generalization to the pediatric population might not be appropriate. Data describing the surgical experience in children with IE are sparse, and, as such, no pediatric-specific surgical indications have been published to date. It is notable that some centers, particularly those in developing countries where IE is more common, have adopted a strategy of aggressive, early intervention (Drs Parvathi and Krishna Iyer, personal communication, 2010).

Early surgical intervention during the acute phase of infection has been associated with good outcomes in adults. $^{13-16}$ It is also very likely that optimal surgical timing is likely crucial for improved outcomes in children. However, to our knowledge, no data have examined the effect of surgical timing on the outcomes in children.

To better understand the surgical indications, timing, and outcomes of children with IE, we aimed to describe the 15-year surgical experience at a large, tertiary-care children's hospital.

\section{METHODS}

\section{Patient Selection and Variables}

We conducted a retrospective review of the pediatric cardiology and cardiovascular surgery databases to identify patients diagnosed with IE at 


\section{Abbreviations and Acronyms \\ IE = infective endocarditis \\ $\mathrm{IQR}=$ interquartile range}

Texas Children's Hospital from January 1996 to December 2010. The Baylor College of Medicine institutional review board approved the study.

All available medical records, laboratory results, imaging reports, and surgical reports were reviewed. All patients 21 years old or younger who were diagnosed with definitive IE, as outlined by the modified Duke criteria, ${ }^{17}$ were included. In cases of recurrent IE, only the index case was included in the present analysis. A history of congenital heart disease, previous cardiac surgery, or the presence of prosthetic graft material was not considered a criterion for exclusion. To improve subject homogeneity, patients with probable endocarditis, as outlined by the modified Duke criteria, ${ }^{17}$ were not included in the present study.

\section{Definitions}

The patients were categorized for analysis into either the medical or surgical group according to the need for surgery as a part of their IE treatment regimen. The surgical group was defined by the need for surgery for acute IE within 6 weeks of starting appropriate intravenous antimicrobial therapy.

Patients with congenital heart defects were grouped into 3 categories: lesions with left to right shunting, lesions affecting the right ventricular outflow tract or requiring the creation of a right ventricle to pulmonary artery conduit for repair, and lesions affecting the left ventricular outflow tract, including bicuspid aortic valve.

Estimation of valvular insufficiency and cardiac function were taken directly from the echocardiographic data. The degree of valvular insufficiency was qualitatively determined by the reporting cardiologist. Ventricular dysfunction was quantitatively defined as an ejection fraction less than $55 \%{ }^{18}$ or a shortening fraction less than 2 Z-scores for age and size when the ejection fraction was not available.

\section{Statistical Analysis}

The data were not normally distributed. Descriptive statistics are reported using the median and interquartile range (IQR) for scale variables and proportions for categorical variables. Univariate analysis of categorical variables was performed using the Pearson chi-square test or Fisher's exact test, as appropriate. Continuous variables were analyzed using the MannWhitney $U$ test. The multivariate factors associated with surgical timing were determined by binary logistic regression analysis and are expressed as odds ratios with $95 \%$ confidence intervals. All survival analyses were performed using Cox regression analysis and are expressed as hazard ratios and $95 \%$ confidence intervals. Kaplan-Meier survival analysis was used to determine the 1-, 5-, and 10-year survival. The 2-tailed test of significance was used in all statistical analyses.

\section{RESULTS \\ Patient Population}

A total of 76 patients at a median age of 8.3 years (IQR, 2.1-14.4 years) who met the inclusion criteria were included in the present study. Of these 76 patients, $46(60.5 \%)$ underwent surgery as a part of their treatment regimen for acute IE. The patient demographics are listed in Table 1. Although no age or racial differences were found between the 2 groups, those in the surgical group were more likely to be male $(P=.016)$.

\section{Infection Site}

The infection site was identified in 23 nonsurgical $(76.7 \%)$ and 46 surgical $(100 \%)$ patients. Native valve involvement $(43 \%$ vs $83 \%, P=.020)$ and left-sided lesions $(27 \%$ vs $54 \%, P=.001)$ were more common in the surgical group. The presence of congenital heart disease was not significantly different between the 2 groups $(P=.172)$. The specific infection sites are listed in Table 2.

\section{Causative Organisms}

Positive culture data were obtained from 46 patients (60.5\%): 17 nonsurgical (56.7\%) and 29 surgical $(63.0 \%)$ patients. Staphylococcus aureus and Streptococcus infections were the most common isolated organisms, occurring in $18(23.7 \%)$ and $17(22.4 \%)$ patients, respectively. Of those with $S$ aureus IE, methicillin-sensitive $S$ aureus was present in 13 patients and methicillinresistant $S$ aureus was present in 5. In the subgroup analysis, $S$ aureus was the most common organism in the surgical group (3\% vs $37 \%, P \leq .001)$ and Streptococcus occurred more frequently in the nonsurgical group (33\% vs $15 \%, P=.019)$. No significant differences were seen in the rates of coagulase-negative Staphylococcus infection $(13 \%$ vs $9 \%, P=.270)$. Other organisms included Escherichia coli in 1 surgical patient (2.2\%), Serratia marcescens in 1 nonsurgical patient $(3.3 \%)$, and Entercoccus faecalis in 1 nonsurgical patient $(3.3 \%)$.

\section{Surgical Data}

The most common indication for surgery was acute, severe valvular insufficiency ( 22 patients, $47.9 \%$ ). Of those, 9 patients $(40.9 \%)$ also had evidence of concomitant ventricular dysfunction. Septic embolization was present in 20 patients $(43.5 \%)$ at surgery and was the primary surgical indication in 12 patients $(26.1 \%)$. Of those 12 patients, cerebrovascular embolization occurred in 10, pulmonary embolization in 1, and renal embolization in 1. Persistent intracardiac vegetation, despite appropriate medical management, was present in 12 patients $(26.1 \%)$. In these patients, the vegetations involved the tricuspid valve with less than severe insufficiency in 5, the right ventricle with less than severe tricuspid insufficiency in 4 , the right atrium with persistent positive blood culture findings in 2, and the right atrium with evidence of inferior vena cava obstruction in 1 . Vegetations were documented in 39 patients $(51.3 \%)$, with $25(64.1 \%)$ having a vegetation of $10 \mathrm{~mm}$ or greater and $14(35.9 \%)$ having a vegetation less than $10 \mathrm{~mm}$. The differences in disease characteristics between the 2 groups are listed in Table 3.

\section{Timing of Surgery}

The median interval to surgery was 3.0 days (IQR, 1.0-7.5 days) from the diagnosis of IE. Although most 
TABLE 1. Patient demographics

\begin{tabular}{lccc}
\hline \multicolumn{1}{c}{ Demographic } & $\begin{array}{c}\text { Nonsurgical } \\
(\mathbf{n}=\mathbf{3 0})\end{array}$ & $\begin{array}{c}\text { Surgical } \\
(\mathbf{n}=\mathbf{4 6})\end{array}$ & $\boldsymbol{P}$ value \\
\hline Age (y) & & & NS \\
$\quad$ Median & 7.0 & 8.7 & \\
IQR & $0.4-16.1$ & $4.6-13.4$ & \\
Male gender (n) & $14(46.7)$ & $34(73.9)$ & .016 \\
Race (n) & & & NS \\
Asian & $1(3.3)$ & $1(2.2)$ & \\
Black & $3(10.0)$ & $7(15.2)$ & \\
Hispanic & $9(30.0)$ & $20(43.5)$ & \\
White & $14(46.7)$ & $18(39.1)$ & \\
Other & $3(10.0)$ & $0(0)$ & \\
Congenital heart disease (n) & $21(70.0)$ & $25(54.3)$ & NS \\
Shunt lesion & $8(38.1)$ & $13(52.0)$ & \\
RVOT lesion & $6(28.6)$ & $3(12.0)$ & \\
LVOT lesion & $5(23.8)$ & $8(32.0)$ & \\
AVV lesion & $2(9.5)$ & $1(4.0)$ & \\
Previous cardiac surgery (n) & $11(36.7)$ & $8(17.4)$ & NS \\
\hline
\end{tabular}

Data in parentheses are percentages. $I Q R$, Interquartile range; $N S$, not significant; RVOT, right ventricular outflow tract lesion; LVOT, left ventricular outflow tract lesion; $A V V$, atrioventricular valve

patients $(35,76 \%)$ underwent surgery within 7 days of the diagnosis, a significant number $(25,54 \%)$ underwent surgery within 3 days. The factors associated with surgery within 3 days from diagnosis are listed in Table 4. On multivariate analysis, infection due to $S$ aureus was a significant factor (odds ratio, $4.8 ; 95 \%$ confidence interval, 1.3-17.8) for surgery within 3 days from diagnosis.

\section{Follow-up and Outcomes}

The median patient follow-up period for the entire cohort was 4.5 years (IQR, 1.3-7.1 years). No significant difference was seen in the median follow-up between the nonsurgical

TABLE 2. Infection sites

\begin{tabular}{lccc}
\hline \multicolumn{1}{c}{ Site } & $\begin{array}{c}\text { Nonsurgical } \\
(\mathbf{n}=\mathbf{3 0})\end{array}$ & $\begin{array}{c}\text { Surgical } \\
(\mathbf{n}=\mathbf{4 6})\end{array}$ & $\boldsymbol{P}$ value \\
\hline Native valve & $13(43.3)$ & $38(82.6)$ & .020 \\
$\quad$ Tricuspid & $7(23.3)$ & $14(30.4)$ & NS \\
Mitral & $4(13.3)$ & $11(23.9)$ & NS \\
Aortic & $1(3.3)$ & $7(15.2)$ & NS \\
Mitral plus aortic & $0(0)$ & $4(8.7)$ & NS \\
Mitral plus tricuspid & $1(3.3)$ & $2(4.3)$ & NS \\
Right atrium & $3(10.0)$ & $3(6.5)$ & NS \\
RVOT & $0(0)$ & $1(2.2)$ & NS \\
Left atrium & $1(3.3)$ & $0(0)$ & NS \\
Left ventricle & $2(6.7)$ & $0(0)$ & .029 \\
Ascending aorta & $0(0)$ & $1(2.2)$ & NS \\
RV-PA conduit valve & $3(10.0)$ & $3(6.5)$ & NS \\
Prosthetic graft & $1(3.3)$ & $0(0)$ & NS \\
Unknown & $7(23.3)$ & $0(0)$ & NS \\
\hline Data presented as $n(\%) . N S$, Not significant; $R V O T$, right ventricular outflow tract; \\
$R V$-PA, right ventricle to pulmonary artery. & &
\end{tabular}

TABLE 3. Disease characteristics

\begin{tabular}{lccc}
\hline \multicolumn{1}{c}{ Characteristic } & $\begin{array}{c}\text { Nonsurgical } \\
(\mathbf{n = 3 0 )}\end{array}$ & $\begin{array}{c}\text { Surgical } \\
(\mathbf{n = 4 6 )}\end{array}$ & $\boldsymbol{P}$ value \\
\hline Left-sided vegetation & $8(26.7)$ & $25(54.3)$ & .001 \\
Any valvular involvement & $16(53.3)$ & $41(89.6)$ & NS \\
Valvular insufficiency & & & \\
$\quad$ None or trivial & $2(6.7)$ & $0(0)$ & .021 \\
$\quad$ Mild & $5(16.7)$ & $12(26.1)$ & NS \\
$\quad$ Moderate & $7(23.3)$ & $12(26.1)$ & NS \\
$\quad$ Severe & $2(6.7)$ & $17(37.0)$ & .037 \\
Presence of ventricular dysfunction & $0(0)$ & $9(19.6)$ & .010 \\
Staphylococcus aureus & $1(3.3)$ & $17(37.0)$ & $<.001$ \\
Septic embolization & $1(3.3)$ & $20(43.5)$ & $<.001$ \\
Vegetation size $\geq 10$ mm & $2(6.7)$ & $23(50.0)$ & $<.001$ \\
\hline Data presented as $\mathrm{n}(\%) . N S$, Not significant. & &
\end{tabular}

group (median, 5.2 years; IQR, 2.8-7.9 years) and surgical group (median, 3.8 years; IQR, 1.1-6.5 years; $P=.095$ ).

The all-cause mortality of the entire cohort during the follow-up period was $7.9 \%$ (6 patients). Although overall mortality was lower in the surgical group $(6.5 \%$ vs $10.0 \%)$, this difference did not reach statistical significance $(P=.583)$. The 1-, 5-, and 10-year survival of the nonsurgical group was $96 \% \pm 4 \%, 89 \% \pm 7 \%$, and $81 \% \pm 10 \%$, respectively. Comparatively, survival among the surgical group was $98 \% \pm 2 \%, 90 \% \pm 8 \%$, and $81 \% \pm 11 \%$ at 1,5 , and 10 years, respectively (log-rank $P=.866$; Figure 1).

A single 30-day operative death $(2.1 \%)$ occurred in a 15-year-old boy with mitral valve IE. He had been undergoing active chemotherapy for acute lymphocytic leukemia when he acutely experienced a catastrophic neurologic insult due to septic cerebral embolization. He arrived at our institution in a moribund state but was taken to the operating room on admission day 2 in an effort to remove any additional source of embolization. However, he was declared brain dead on admission day 11 , despite all resuscitative measures.

Of the remaining 5 deaths, 2 were associated with IE. An infant with a structurally normal heart experienced worsening mitral valve insufficiency secondary to IE. Because of the patient's size at the development of infection, immediate surgical therapy was deferred. The patient successfully completed a 6-week antibiotic course but died 8 months

TABLE 4. Univariate factors associated with surgery within 3 days of diagnosis of infective endocarditis

\begin{tabular}{|c|c|c|}
\hline Factor & OR & $\mathbf{9 5} \% \mathrm{CI}$ \\
\hline Presence of ventricular dysfunction & 5.1 & $1.1-22.3$ \\
\hline Staphylococcus aureus infection & 4.7 & $1.3-16.9$ \\
\hline Left-sided lesion & 2.0 & $0.7-5.4$ \\
\hline Septic embolization & 1.9 & $0.6-5.4$ \\
\hline Valvular involvement & 1.6 & $0.4-6.8$ \\
\hline Severe valvular insufficiency & 1.2 & $0.4-3.8$ \\
\hline
\end{tabular}

$O R$, Odds ratio; $C I$, confidence interval. 


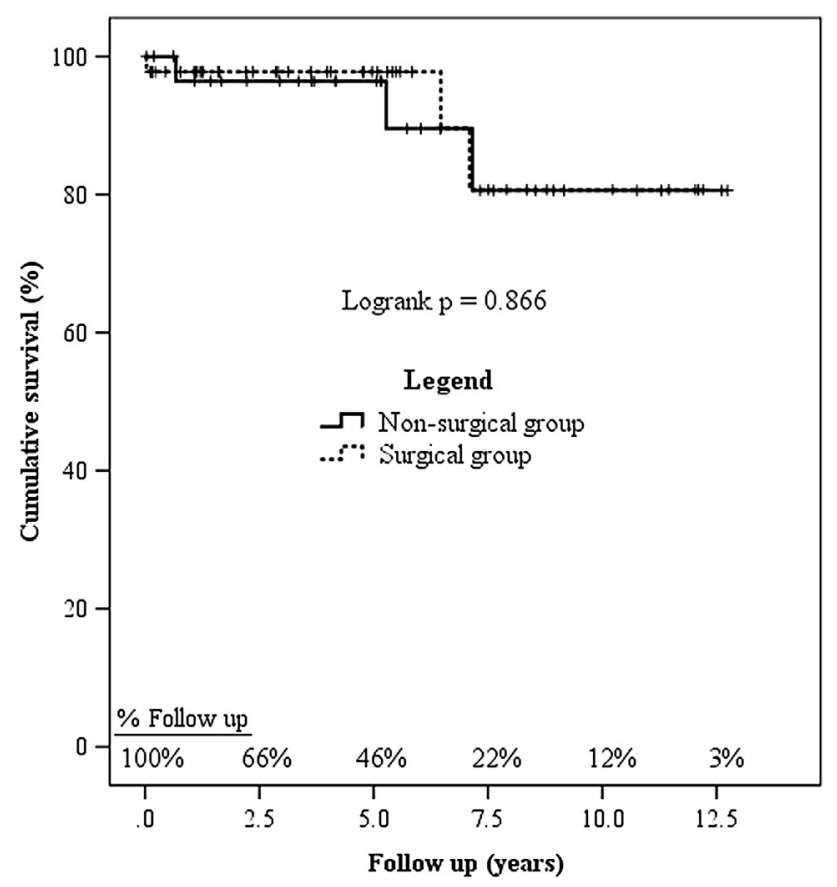

FIGURE 1. Kaplan-Meier survival function stratified by treatment group.

later of complications from congestive heart failure as a valvular surgical evaluation was being performed. The second IE-related mortality occurred in a surgical patient 7.1 years after aortic valve replacement owing to an initial episode of IE. He presented with IE of the prosthetic aortic valve but succumbed to overwhelming sepsis soon after presentation.

No evidence of postoperative septic embolization was found. The recurrence rate of IE was not significantly different statistically, occurring in 2 nonsurgical patients $(6.7 \%)$ and 1 surgical patient $(2.2 \% ; P=.330)$. The recurrence of IE was significantly associated with increased mortality (hazard ratio, 6.4; 95\% confidence interval, 1.1-36.3).

Of the 38 surgical patients with native valve involvement, $19(50.0 \%)$ required valve replacement and 19 underwent successful native valve repair. Of the 19 patients requiring valve replacement, 12 involved the aortic position, 5 the mitral position, and 2 the right ventricular outflow tract. In the aortic position, an aortic homograft was used in 8 patients, a pulmonary autograft (Ross procedure) in 3, and a bioprosthetic valve in 1 . In the mitral position, a mechanical valve was used in 4 patients and a composite valve in 1 . In the right ventricular outflow tract, a valved conduit was used in both patients. Valve replacement was not significantly associated with mortality $(P=.435)$. Although no repeat valve replacements occurred in the surgical group, 3 nonsurgical patients $(10.0 \%)$ required valve replacement $(P=.029)$ within the follow-up period after completing their medical therapy course.

No significant differences were seen in mortality ( $P=.562)$ or overall valve replacement $(P=.469)$ between patients who had surgery more than 7 days versus within 3 days from diagnosis.

\section{DISCUSSION}

The continuously changing epidemiology and characteristics of pediatric IE demands an evolving understanding of treatment options and effect on outcomes to improve associated morbidity and mortality. In the present study, 1 of the largest reported pediatric surgical cohorts for IE, we found that early surgery can be successfully performed in children with high rates of native valve repair and low rates of recurrence and mortality.

Although no pediatric-specific surgical guidelines for acute IE exist, established American Heart Association adult guidelines have been endorsed by the American Academy of Pediatrics. ${ }^{12}$ We found that the indications for surgery at our institution generally mirror those identified in adult guidelines, including ventricular dysfunction, acute valvular insufficiency, and uncontrolled infection. These findings are similar to a recent, multicenter review describing the surgical indications of children with congenital heart disease who developed IE. ${ }^{19}$ In addition, we found a number of other factors associated with surgical intervention, including the presence of left-sided vegetations, $S$ aureus infection, and a history of at least 1 previous embolization event. These factors might represent an institutional bias toward an aggressive surgical approach to minimize embolization risk owing to a high incidence of $S$ aureus infection in our cohort. However, additional appropriately designed comparative studies would need to be conducted to adequately evaluate the suitability of these indications.

Recent studies of adult populations have demonstrated improved survival when surgery for IE was performed early in specific patient subgroups. ${ }^{13-15}$ This benefit has been attributed to several factors, including the early management of a reversible cause of ventricular dysfunction, minimizing embolization risk, and aggressive treatment of $S$ aureus. This is an important consideration owing to the highly virulent nature of $S$ aureus, because these children are more likely to present with significantly advanced disease, including aortic root involvement, valvular damage, and abscess formation. In addition, changing trends in the epidemiology have shown that $S$ aureus has become 1 of the most common pathogens responsible for $\mathrm{IE}^{20,21}$ which was reflected in our findings. Owing to the risk of advanced disease, our institutional practice is to take children with $S$ aureus-related IE to the operating room as soon as possible with the goal of minimizing the risk of disseminated disease and septic embolization and improving the possibility of native valve repair.

To date, information is limited concerning the effects of surgical timing on the outcomes in children. Although Nomura and colleagues ${ }^{7}$ demonstrated that surgical intervention during the active phase of infection might be 
a necessary adjunct to antibiotic therapy in certain subgroups, the timing of the surgical intervention was not described. Recently, Hickey and colleagues ${ }^{5}$ demonstrated that surgery occurring within 1 week of diagnosis of a cerebrovascular accident due to septic embolization was safe. However, that study was limited by the small patient population in the cerebrovascular accident group and lacked a description of the interval to surgery from the diagnosis of IE.

In the present study, we found that most surgical patients underwent surgery within 7 days of diagnosis of IE and that one half of those patients underwent surgery within 3 days. Surgery within 3 days was significantly associated with ventricular dysfunction or $S$ aureus infection on univariate analysis and $S$ aureus infection on multivariate analysis. As a result of our aggressive surgical approach, $50 \%$ of patients underwent native valve repair, no postoperative embolization events occurred, and mortality was low.

Previous reports have described greater morbidity and mortality in patients with ventricular dysfunction or $S \mathrm{au}$ reus infection. ${ }^{21}$ Thus, early surgical involvement in these children might have an advantageous role in their treatment by minimizing the risk of embolization and abscess formation in the case of staphylococcal infection and potentially reverse the cause of ventricular dysfunction. We have demonstrated early surgery can be safely performed in children, with low postoperative mortality and acceptable mediumto long-term outcomes.

\section{Study Limitations}

Although the present study is 1 of the largest pediatric surgical cohorts with regard to IE, it was limited by its single-center retrospective design of a moderate number of patients. Although all available data were used in the statistical analyses, the negative endpoints described in the present study might have resulted from a lack of statistical power. Therefore, it would be difficult to exclude the possibility of a type II error, and additional studies with greater numbers of patients are required to establish negative associations.

The design of the present study prevented a direct comparison between the surgical and nonsurgical groups owing to an inherent selection bias. Attempts to alleviate this bias using statistical methods, such as propensity scoring, were not possible owing to the small study size. Thus, the findings of the present study should be interpreted in the context of the descriptive nature of the data. Although the present study was not designed to directly compare the outcomes of early surgery with those of late surgery or medical therapy alone, it has demonstrated that early surgery can be performed safely and effectively in children. Finally, significant associations with respect to surgical indications and timing represent the practice of our large, US tertiarycare children's hospital and might not be generalized to dissimilar institutions or locations.

\section{CONCLUSIONS}

Early surgical intervention in children with acute IE can be performed safely with excellent results. The benefit of early surgery might play a significant role in the treatment of IE caused by $S$ aureus by minimizing the risk of progressive cardiac disease and septic embolization. Furthermore, early surgery, in conjunction with appropriate antibiotic therapy, can be performed in children safely, with excellent results, even in the acute phase of infection. Additional study is required to better understand the overall indications for surgery and those who might benefit most from early surgical intervention.

\section{References}

1. Day MD, Gauvreau K, Shulman S, Newburger JW. Characteristics of children hospitalized with infective endocarditis. Circulation. 2009;119:865-70.

2. Coward K, Tucker N, Darville T. Infective endocarditis in Arkansan children from 1990 through 2002. Pediatr Infect Dis J. 2003;22:1048-52.

3. Rosenthal LB, Feja KN, Levasseur SM, Alba LR, Gersony W, Saiman L. The changing epidemiology of pediatric endocarditis at a children's hospital over seven decades. Pediatr Cardiol. 2010;31:813-20.

4. Delmo Walter EM, Musci M, Nagdyman N, Hubler M, Berger F, Hetzer R. Mitral valve repair for infective endocarditis in children. Ann Thorac Surg. 2007;84: 2059-65.

5. Hickey EJ, Jung G, Manlhiot C, Sakopoulos AG, Caldarone CA, Coles JG, et al. Infective endocarditis in children: native valve preservation is frequently possible despite advanced clinical disease. Eur J Cardiothorac Surg. 2009;35: $130-5$.

6. Karaci AR, Aydemir NA, Harmandar B, Sasmazel A, Saritas T, Tuncel Z, et al. Surgical treatment of infective valve endocarditis in children with congenital heart disease. J Card Surg. 2012;27:93-8.

7. Nomura F, Penny DJ, Menahem S, Pawade A, Karl TR. Surgical intervention for infective endocarditis in infancy and childhood. Ann Thorac Surg. 1995;60: 90-5.

8. Vikram HR, Buenconsejo J, Hasbun R, Quagliarello VJ. Impact of valve surgery on 6-month mortality in adults with complicated, left-sided native valve endocarditis: a propensity analysis. JAMA. 2003;290:3207-14.

9. Niwa K, Nakazawa M, Tateno S, Yoshinaga M, Terai M. Infective endocarditis in congenital heart disease: Japanese national collaboration study. Heart. 2005;91: 795-800.

10. Weber R, Berger C, Balmer C, Kretschmar O, Bauersfeld U, Pretre R, et al. Interventions using foreign material to treat congenital heart disease in children increase the risk for infective endocarditis. Pediatr Infect Dis J. 2008;27: 544-50.

11. Yoshinaga M, Niwa K, Niwa A, Ishiwada N, Takahashi H, Echigo S, et al. Risk factors for in-hospital mortality during infective endocarditis in patients with congenital heart disease. Am J Cardiol. 2008;101:114-8.

12. Baddour LM, Wilson WR, Bayer AS, Fowler VG Jr, Bolger AF, Levison ME, et al. Infective endocarditis: diagnosis, antimicrobial therapy, and management of complications: a statement for healthcare professionals from the Committee on Rheumatic Fever, Endocarditis, and Kawasaki Disease, Council on Cardiovascular Disease in the Young, and the Councils on Clinical Cardiology, Stroke, and Cardiovascular Surgery and Anesthesia, American Heart Association: endorsed by the Infectious Diseases Society of America. Circulation. 2005;111:e394-434.

13. Bishara J, Leibovici L, Gartman-Israel D, Sagie A, Kazakov A, Miroshnik E, et al. Long-term outcome of infective endocarditis: the impact of early surgical intervention. Clin Infect Dis. 2001;33:1636-43.

14. Kim DH, Kang DH, Lee MZ, Yun SC, Kim YJ, Song JM, et al. Impact of early surgery on embolic events in patients with infective endocarditis. Circulation. 2010;122(11 Suppl):S17-22.

15. Lalani T, Cabell CH, Benjamin DK, Lasca O, Naber C, Fowler VG Jr, et al. Analysis of the impact of early surgery on in-hospital mortality of native valve endocarditis: use of propensity score and instrumental variable methods to adjust for treatment-selection bias. Circulation. 2010;121:1005-13.

16. Kang DH, Kim YJ, Kim SH, Sun BJ, Kim DH, Yun SC, et al. Early surgery versus conventional treatment for infective endocarditis. $N$ Engl J Med. 2012;366: 2466-73. 
17. Li JS, Sexton DJ, Mick N, Nettles R, Fowler VG Jr, Ryan T, et al. Proposed modifications to the Duke criteria for the diagnosis of infective endocarditis. Clin Infect Dis. 2000;30:633-8.

18. Lang RM, Bierig M, Devereux RB, Flachskampf FA, Foster E, Pellikka PA, et al. Recommendations for chamber quantification: a report from the American Society of Echocardiography's Guidelines and Standards Committee and the Chamber Quantification Writing Group, developed in conjunction with the European Association of Echocardiography, a branch of the European Society of Cardiology. J Am Soc Echocardiogr. 2005;18:1440-63.
19. Murakami T, Niwa K, Yoshinaga M, Nakazawa M. Factors associated with surgery for active endocarditis in congenital heart disease. Int J Cardiol. 2012;157 59-62.

20. Alshammary A, Hervas-Malo M, Robinson JL. Pediatric infective endocarditis: has Staphylococcus aureus overtaken viridans group streptococci as the predominant etiological agent? Can J Infect Dis Med Microbiol. 2008;19:63-8.

21. Selton-Suty C, Celard M, Le Moing V, Doco-Lecompte T, Chirouze C, Iung B, et al. Preeminence of Staphylococcus aureus in infective endocarditis: a 1-year population-based survey. Clin Infect Dis. 2012;54:1230-9. 